\title{
Associative and Implicit Memory Performance as a Function of Cognitive Reserve in Elderly Adults with and without Mild Cognitive Impairment
}

\author{
Salvador Algarabel ${ }^{1}$, Alicia Sales ${ }^{1}$, Alfonso Pitarque ${ }^{1}$, Juan C. Meléndez ${ }^{1}$, Joaquín Escudero ${ }^{2}$ and \\ Teresa Mayordomo ${ }^{1}$ \\ 1 Universidad de Valencia (Spain) \\ ${ }^{2}$ Hospital General de Valencia (Spain)
}

\begin{abstract}
This study aims to analyze implicit and explicit memory performance as a function of cognitive reserve (CR) in a healthy control group $(N=39)$ and a mild cognitive impairment $(\mathrm{MCI})$ group $(N=37)$. Both groups were subdivided into high and low cognitive reserve, and were asked to complete an explicit and implicit associative recognition tasks. The results showed that the control group was able to learn both tasks $\left(\eta^{2}=.19, p<.0001\right)$, and the high CR group fared better $\left(\eta^{2}=.06, p<.05\right)$. The MCI sample, conversely, was unable to learn the implicit relationship, and showed very little learning on the explicit association task. Participants diagnosed with $\mathrm{MCI}$ showed little plasticity in learning associations regardless of $\mathrm{CR}\left(\eta^{2}=.12, p<.01\right)$.
\end{abstract}

Received 16 April 2015; Revised 12 August 2015; Accepted 25 August 2015

Keywords: cognitive reserve, mild cognitive impairment, associative recognition, explicit memory, implicit memory.

Not all types of memory decline with age in the same way. Comparisons of healthy young and old people have revealed that explicit memory deteriorates considerably by age, whereas performance on so-called implicit memory tasks based on familiarity declines less or even remains intact (Dew, Bayen, \& Giovanello, 2007; Fleischman, Wilson, Gabrieli, Bienias, \& Bennett, 2004; Light \& Singh, 1987). These effects have occurred when outcomes on tasks like free recall or recognition (explicit tasks) were compared to others such as fragment completion or priming tasks (implicit tasks).

Within episodic memory, associative memory is the most affected by age, followed by memory for item information (Old \& Naveh-Benjamin, 2008). To explain impaired memory with age, Naveh-Benjamin and his colleagues (Bender, Naveh-Benjamin, \& Raz, 2010; Old \& Naveh-Benjamin, 2008) formulated the associative deficit hypothesis according to which elderly adults are more deficient at binding pieces of information together than at recalling the information itself, but both deteriorate with age. In light of the research findings to date, it is logical that explicit tests of relational memory show pronounced deficit with age, as they require learning and retrieval of explicit associative relationships (e.g. Dew et al., 2007; Dew \& Giovanello, 2010). The present research is particularly interested in

Correspondence concerning this article should be addressed to Alfonso Pitarque. Universidad de Valencia - Methodology-Psychology. Valencia (Spain).

E-mail: pitarque@uv.es analyzing performance on tasks where respondents must associate different pieces of verbal information, rather than associate an item and its context. In a largescale meta-analysis, Old \& Naveh-Benjamin (2008) concluded that associative memory was more deficient than item information memory, and more on recognition tasks than in free recall. An important point raised in that meta-analysis is that "intentionality" plays a role in the extent of this deficit, which contradicts the ongoing controversy about whether or not elderly people show deficits on priming or similar implicit memory tasks. In a priming paradigm, participants are tested on the second of two tasks as experimenters try to detect an effect from the first one; of course participants are not made aware of any relationship between the two. Most studies have found no drop in performance in priming tasks with age (Dew et al., 2007; Fleischman et al., 2004; Light \& Singh, 1987), but in some cases, a small decrease has been observed on conceptual priming tasks (Dew et al., 2007; Fleischman \& Gabrieli, 1998 for a review). Given the methodological difficulty of stating that null differences occurred in performance on these very low-difficulty tasks, it is conceivable that contradictory results be found.

The next step in exploring implicit memory deficit is to test implicit associative memory. It was once believed that establishing implicit relationships between novel pieces of information was impossible without elaborative processing, considered a necessary precondition (Dew et al., 2007). However, further research has shown that relational priming can occur through 
non-elaborative processing (e.g. Reingold \& GroshenGottstein, 1996) using perceptual as well as conceptual dimensions (e.g. Graf \& Schacter, 1985). Similarly experiments on implicit learning show that implicit relationships between items can be established without awareness (e.g. Reber, Walkenfeld, \& Hernstadt, 1991). Generally speaking, older adults can formulate implicit relationships. Whether or not a deficit by age is detected may depend on the sort of association being made (Light \& Singh, 1987).

Age and other variables modulate elderly performance on memory tasks. In particular, cognitive reserve (CR) is known to act as a moderator, influencing performance in elderly adults. Cognitive reserve is a person's ability to cope with age-related deterioration in the brain processes underlying behavior (Stern, 2009). It has been widely used to explain the disparity between brain damage and behavioral manifestations of Alzheimer's disease (AD) or similar types of dementia. Brain autopsies have shown that some people with extensive brain damage on par with AD patients behaved "normally" in their lifetimes and were never diagnosed with the illness (Stern, 2009). People with high CR are somehow able to compensate for cognitive deficit occurring as a function of age or illness. Several studies of normal aging have found that people with high levels of education generally maintain greater cognitive functioning and have less risk of developing symptoms of dementia as they age (Angel, Fay, Bouazzaoui, Baudouin, \& Isingrini, 2010; Vance \& Crowe, 2006; but see Zahodne et al., 2011). Likewise, a review by Valenzuela and Sachdev (2006) showed that based on combined odds ratio, people with a history of high occupational status had less risk of incident dementia than low-status individuals after controlling for numerous covariates, as other studies have done (Scarmeas \& Stern, 2003; Vance \& Crowe, 2006). Some studies have suggested that IQ estimates -or premorbid IQ estimates- may actually be stronger measures of cognitive reserve. In that vein, Solé-Padullés et al. (2009) reported a clear relationship between gray matter and cognitive reserve. Similarly, Colom, Jung, and Haier (2006) found a positive correlation between increased gray matter and certain measures, including the WAIS vocabulary subtest. However CR is a hypothetical construct that cannot be measured directly. Proxy variables and latent variable models have been used in an attempt to operationalize it. The criteria currently used to measure CR used a factor score combining education, occupation, leisure activities, and IQ, all variables associated with cognitive ability that indirectly tap cognitive reserve (Solé-Padullés et al., 2009). To postulate an association between intellectual capacity and memory performance is nothing new (e.g., Conway, Kane, \& Engle, 2003). Therefore, it would be no surprise to find that cognitive reserve and explicit memory are connected. Accordingly, more highly educated older adults can implement alternative cognitive strategies to cope with age-related changes.

It would be harder to postulate a link between cognitive reserve and implicit memory, however. We only know one study that analyzed that relationship (Gordon, Soldan, Thomas, \& Stern, 2013). This study examines repetition priming, that is, changes in one's ability to detect a stimulus that was previously presented. Given the lack of strong data about relational implicit and explicit memory and cognitive reserve, the present research was designed to study the relationship between cognitive reserve, memory performance (explicit-implicit), and mental status (healthy vs. mild cognitive impairment). Based on the literature, we expect to find a strong connection between cognitive reserve and people's ability to deduce explicit relationships between pieces of information. However, since the relationship between intellectual ability and automatic memory is weak or non-existent (e.g. Reber et al., 1991) and the effect of cognitive reserve decreases with $\mathrm{MCI}$ diagnosis, we expect those variables to have less impact on people's ability to form implicit relationships. Furthermore, given that explicit relational memory is more affected by age than implicit, we expect to observe that one develops to compensate for the other if given the opportunity to do so.

Whereas Gordon et al. (2013) analyzed performance on a purely implicit task, this paper examines how well people draw implicit relationships during an explicit study task. We modified the design of an associative recognition paradigm so that respondents had to learn a conceptual explicit relationship between the two words in a pair of stimuli as well as a perceptual implicit relationship, based on the rule that the two words share the same vowel letter (i.e. the letter "a") but not another vowel (i.e. the letter "o"; see Algarabel et al., 2009; Algarabel et al., 2012; Algarabel \& Pitarque, 2010; Algarabel, Pitarque, Cómbita, \& Rodríguez 2013; Algarabel, Pitarque, Tomás, \& Mazón, 2010; Algarabel, Rodríguez et al., 2010). Relative to a traditional test condition, this gives an index of how participants differentially make associations explicitly versus implicitly. In that vein our experiment is similar to a classical "levels-of-processing" experiment (Craik, 2002; Craik \& Tulving, 1975), in which two different ways of encoding information are usually confronted (deep or semantic vs shallow or perceptual). Since in our experiment we manipulate a condition of semantic processing against another condition of perceptual plus semantic processing, we expect to find a better recognition in this later condition than in the former one, as it would be predicted by the "levels-of-processing" theory (Craik, 2002; Craik \& Tulving, 1975). 
Finally, as described above, in addition to studying performance in healthy and MCI elderly adults, we will also examine the $C R$ variable. It is well established that people with high CR use mental processes to compensate when a task requires brain circuits that have deteriorated with age. Given that implicit and explicit memory use different cognitive resources, we will investigate the possibility that people with high $\mathrm{CR}$ benefit more from making implicit associations, using implicit memory to boost explicit memory.

\section{Method}

\section{Participants}

The total sample was comprised of 76 participants. The MCI group was composed of 37 patients ( 25 women, 12 men, mean age $=74.95$ years) recruited from the Department of Neurology of the Hospital General of Valencia, Spain. The healthy control subjects were 39 cognitively normal volunteers ( 29 women, 10 men, mean age $=69.75$ years), recruited from centers specializing in elderly care in the city of Valencia, Spain (see demographic data in Table 1).
Diagnosis was the end-result of an extended neuropsychological examination applied to both the MCI sample and the healthy sample (see Table 1 for detailed data). Psychometric evaluation included the MiniMental State Examination (MMSE; Folstein, Folstein, \& McHugh, 1975; Spanish version, Lobo, Saz, \& Marcos, 2002), and the Global Deterioration Scale (GDS; Reisberg, Ferris, de Leon, \& Crook, 1982). Symptoms of depression were assessed by Yesavage's Geriatric Depression Scale (Yessavage et al., 1983). More cognitively oriented abilities were also evaluated. In particular, participants completed a category recall and a verbal fluency subtest of the Revised Barcelona Test (TBR, Peña-Casanova, 2005). Other memory dimensions were evaluated with the Logical Memory Immediate subtest and forward and Backward Digit Span (Wechsler Memory Scale III, WMS; Wechsler, 2004), vocabulary (Wechsler Intelligence Scale for Adults-III, WAIS; Wechsler, 2001), the Spain-Complutense Verbal Learning Test (TAVEC; Benedet \& Alejandre, 1998) and visuospatial ability (Rey Complex Figure Test; Rey, 1999). Inclusion criteria for the control group required that participants had neither impairment that interfered with their daily activities, nor depressive symptomatology.

Table 1. Means (and SD) of demographic data and neuropsychological performance as function of groups (Healthy vs MCI: Mild Cognitive Impairment) and cognitive reserve (CR: Low vs High). Note: $a=$ main effect of group; $b=$ main effect of $C R ; c=$ interaction effect

\begin{tabular}{|c|c|c|c|c|c|}
\hline & \multicolumn{2}{|c|}{ Healthy controls } & \multicolumn{2}{|l|}{ MCI } & \multirow[b]{2}{*}{$\begin{array}{l}\text { Significant } \\
\text { contrasts }\end{array}$} \\
\hline & High CR & Low CR & High CR & Low CR & \\
\hline Age & $68.50(5.49)$ & $71.00(7.21)$ & $75.84(5.15)$ & $74.06(7.62)$ & a \\
\hline $\begin{array}{l}\text { Education (Illiteracy, primary, secundary } \\
\text { and university studies) }\end{array}$ & $0: 11: 6: 3$ & $3: 14: 2: 0$ & $3: 8: 4: 4$ & 6:11:1:0 & \\
\hline Sex (male, female) & $6: 14$ & $4: 15$ & $7: 10$ & $5: 15$ & \\
\hline MMSE & $28.65(1.39)$ & $28.29(0.99)$ & $26(2.53)$ & $25.83(1.50)$ & a \\
\hline Global Deterioration Scale (GDS) & $1.35(0.67)$ & $1.26(0.45)$ & $2.68(0.74)$ & $2.4(0.85)$ & a \\
\hline Geriatric Depression Scale (Yesavage) & $7.50(5.02)$ & $9.56(4.11)$ & $7.26(5.17)$ & $11.56(6.36)$ & $\mathrm{b}$ \\
\hline Verbal fluency Phonemic (letter P) (TBR) & $26.95(10.22)$ & $23.78(9.42)$ & $19.50(8.54)$ & $17.53(9.01)$ & a \\
\hline Verbal fluency Semantic (animals) (TBR) & $17.15(6.41)$ & $14.83(4.9)$ & $12.11(4.61)$ & $11.53(3.28)$ & a \\
\hline Praxis-imitation right (TBR) & $8.94(1.68)$ & $9.29(1.10)$ & $9.35(1.11)$ & $8.88(2.08)$ & \\
\hline Praxis-imitation left (TBR) & $9(1.65)$ & $9.33(1.08)$ & $9.35(1.11)$ & $8.88(2.08)$ & \\
\hline Praxis-symbolic (TBR) & $7(1.38)$ & $6.83(1.50)$ & $7.11(1.05)$ & $6.22(1.56)$ & $\mathrm{b}$ \\
\hline Vocabulary Subtest (WAIS) & $30(10.44)$ & $22.79(10.60)$ & $21(11.79)$ & $12.39(5.92)$ & $a, b$ \\
\hline Digit Span Forward (WMS) (total) & $9.8(2.63)$ & $7.36(2.63)$ & $9.61(2.51)$ & $6.63(1.74)$ & $\mathrm{a}$ \\
\hline Digit Span Backward (WMS) & $4.25(1.80)$ & $4.61(1.94)$ & $3.66(1.46)$ & $2.94(1.14)$ & a \\
\hline Logical-Memory Immediate Units (WMS) & $12.10(3.31)$ & $9.57(2.65)$ & $8.05(3.06)$ & $7.3(3.76)$ & $a, b$ \\
\hline Logical-Memory Immediate Themes (WMS) & $10.94(3.26)$ & $10.63(3.80)$ & $8(2.30)$ & $8.2(2.75)$ & a \\
\hline TAVEC-Immediate recall of the first trial & $4.95(1.73)$ & $4.63(1.57)$ & $3.36(2.03)$ & $2.75(1.29)$ & a \\
\hline TAVEC-Immediate recall of the fifth trial & $11.6(3.20)$ & $10.52(3.12)$ & $6.57(2.71)$ & $5.55(3.15)$ & a \\
\hline TAVEC-Learning & $44.6(12.36)$ & $40.73(11.83)$ & $26.84(11.16)$ & $23.3(7.87)$ & $\mathrm{a}$ \\
\hline TAVEC-free long-term memory & $11.15(2.07)$ & $9.3(3.03)$ & $4.94(3.94)$ & $5.26(3.51)$ & $\mathrm{a}, \mathrm{c}(p=.08)$ \\
\hline Visual Memory Test-Copy (Rey) & $30.6(7.41)$ & $27.61(8.05)$ & $24.29(10.23)$ & $19.39(11.94)$ & $\mathrm{a}, \mathrm{b}$ \\
\hline Visual Memory Test- Memory (Rey) & $13.65(6.90)$ & $7.55(8.08)$ & $6.70(6.75)$ & $4.57(7.55)$ & $\mathrm{a}, \mathrm{b}, \mathrm{c}(p=.08)$ \\
\hline
\end{tabular}


Mild Cognitive Impairment was defined according to the diagnostic criteria specified by Petersen (2004). Exclusion criteria for patients were: significant asymptomatic neurovascular alteration disease confirmed by brain MRI, a history of previous symptomatic stroke, any medical condition significantly affecting the brain, serious psychiatric symptoms or a history of drug abuse. All participants gave written informed consent for the study, which was approved by the institutional review boards of the General Hospital of Valencia and the University of Valencia, Spain.

\section{Cognitive reserve groups}

Cognitive reserve measurement was established according to the guidelines put forward in previous studies (Solé-Padullés et al., 2009; Stern, 2009; Stern et al., 2005) taking into account performance in four variables measured by a questionnaire: WAIS-III vocabulary scale (range 1-19); education level (1 = illiteracy; no formal studies, 2 = primary studies, 3 = secondary studies, $4=$ universitary studies); occupation ( $1=$ non-qualified manual, 2 = qualified manual, 3 = qualified non-manual or technician, 4 = professional; university degree required, 5 = manager or director; university degree required), and leisure activities (range 0-14). A principal component factor analysis was carried out on the previous measures (e.g. Solé-Padullés et al., 2009). A single factor solution explained $42.3 \%$ of the variance. The final individual CR score was obtained multiplying each individual CR score times the factor loading. According to this analysis, we formed two groups of low and high cognitive reserve in both, the healthy and the MCI samples, with an average score of 10.88 (range: $8.87-$ 12.93) and 15.40 (range: $12.98-22.05)$, and 8.44 (range: 6.77-9.53) and 12.45 (range: 10.18-17.41), respectively. The four samples were equated in gender and education. The healthy and MCI groups differed in age (means 69.75 and $74.95, t(74)=3.54, p<.01$, respectively) but the two subgroups of the healthy control sample were equated in age (means $71.00 \mathrm{vs} 68.50, t(37)<1)$, as were the MCI subsamples (means 74.06 vs $75.84, t(35)<1$ ), respectively.

\section{Materials and Procedure}

Participants were first evaluated neuropsychologically and then participated in the associative recognition experiment. The experiment was administered in two identical blocks, with a study and a test phase in each, differing only in the stimuli presented for test. Each block consisted of the presentation for study and immediate test of 12 lists of word pairs. Six of lists belonged to the explicit condition (E) and the other 6 to the explicit plus implicit one (E+I). List composition was elaborated with 192 Spanish words, 3-9 letters in length, containing half of them the letter " $a$ " but not the letter "o" (list a-a), and the other half the letter "o" but not "a" (list o-o). Both lists were equated in mean frequency per two millions (Alameda \& Cuetos, 1995) and in number of letters (a-a: 97.37 and 5.6; o-o: 96.15 and 5.34 for frequency and length, respectively). We paired randomly 96 "a-a" words (48 pairs) and 96 "o-o" words (48 pairs). For the study phase no pair was formed crossing an "a" word with an "o" word. In consequence, the 96 pairs of words were grouped into 12 study lists with 8 pairs each, and within each list, four pairs of words containing the letter " $a$ " (e.g. urna-bazar) and four pairs containing the letter "o" (e.g. bisonte-libro).

Participants studied and were tested with the first 6 list under the E condition, and then, studied and were tested in the remaining 6 lists under E+I conditions (counterbalanced across participants). Whereas the study phase of both conditions was identical, it is in the test situation where the difference arose. In the explicit (E) test condition, eight word pairs, 4 left intact, and 4 rearranged (items presented in different pairs at study), were presented. The rearranged pairs had the additional restriction that no two words in a pair were extracted from a different letter set. That is, if a word pair at study was from the "a-a" set, its rearrangement produced also an "a-a" pair. The same restriction applied to "O-O" sets. Response in this case should be based in the retrieval of the original explicitly learned association between the two studied words. In the explicit plus implicit $(E+I)$ test condition rearranged pairs were constructed differently. In this case, the unpaired words were in all cases, of the "a-o" and the "o-a" types. That is, although the intact pairs remained the same, the words in the unpaired condition were "uncoupled" in relation to their letter composition, allowing the evaluation of their effect. The evaluation of this condition relies on the assumption that participants do not become aware of the implicit relation. If so, they could easily achieve a $100 \%$ rejection of the unpaired words and the experiment would not accomplish its purpose. To avoid the possibility that some participants may have acquired partial knowledge of this manipulation throughout the testing phase, they were questioned at the end of the experiment about the strategies they used for responding, and whether they have noticed anything special about the stimuli (Algarabel et al., 2009, 2010a, 2013). None of the participants mentioned anything about the letter composition of the words. Two versions of the test stimuli were formed for the purpose of counterbalancing. Half of the subjects received as unpaired what the other half had received as paired, and vice versa. Study lists consisted in the random presentation of 8 word pairs, 4 seconds each, in a continuous cycle, and was followed 
by a test of 8 word pairs. Four of these test pairs were identical to the study pairs and 4 were rearranged. Participants made a "yes-no" judgment about each pair of stimuli (if kept intact or not from study) using the " $\mathrm{d}$ " and " $\mathrm{k}$ " keys of a computer keyboard. The words were visible until the participant responded, at which moment the following pair appeared. Words were presented in the center of a computer screen in black on a white background, lowercase, Courier New Bold 18 point.

\section{Results}

Given the impossibility of matching age across healthy and MCI samples, data were analyzed by means of mixed analysis of covariance (ANCOVA) of 2 groups (healthy vs MCI; between subjects) x 2 CR (high vs low; between subjects) $\times 2$ tasks (E vs $\mathrm{E}+\mathrm{I}$; within subjects) taking age as covariate and hits minus false alarms (and then these two variables separately) as dependent variables (see table 2).

The mixed ANCOVA $2 \times 2 \times 2$ on hits minus false alarms showed as significant the main effects of both the variable group $\left(F(1,71)=16.41, p<.0001, \eta^{2}=.19\right)$, indicating that the healthy sample performed better than the MCI sample (means 0.27 and 0.12 , respectively), and the variable $\mathrm{CR}\left(F(1,71)=4.19, p<.05, \eta^{2}=\right.$ .06), indicating that the high $C R$ group performed better than the low CR group (means 0.23 and 0.16 , respectively), as expected. The interaction groups by tasks was also significant $\left(F(1,71)=9.43, p<.01, \eta^{2}=.12\right)$. Post-hoc analysis (Bonferroni t-tests) on this interaction showed that the healthy sample performed better the E+I task than the E task (means 0.34 and 0.19, respectively; $t(38)=5.64, p<.0001$ ) whereas there were no differences between these tasks in the MCI group (means 0.10 and 0.13 , respectively; $t(36)<1$ ). Finally the interaction groups by tasks by CR was also significant $\left(F(1,71)=4.14, p<.05, \eta^{2}=.06\right)$. Post-hoc analysis on this interaction showed again that the two healthy groups performed better in the E+I condition than in the E condition, that is, they learned the implicit relations to improve their recognition (means of the high
CR healthy group 0.40 and 0.28 , for the $\mathrm{E}+\mathrm{I}$ and $\mathrm{E}$ tasks, respectively, $t(19)=3.56, p<.01$; means of the low $C R$ healthy group 0.30 and 0.11 , respectively, $t(18)=4.39$, $p<.0001)$. However the two MCI groups performed in a similar way in both tasks, although this difference was marginally significant in the high CR MCI group (means of the high CR MCI group 0.14 and 0.08 , for $\mathrm{E}+\mathrm{I}$ and $\mathrm{E}$ tasks, respectively, $t(18)=1.74, p<.10$; means of the low CR MCI group 0.12 and 0.10 , respectively, $t(17)<1$ ).

Globally taken these results indicate that two healthy groups performed better in the E+I condition than in the $\mathrm{E}$ condition but none of these differences were visible in the MCI group. However, the high CR MCI group tended to take advantage $(p<.10)$ of the implicit manipulation better than the low CR MCI group using better the implicit relations to improve and compensate their baseline level of explicit responding. Finally, to rule out a floor effect on hits minus false alarms (especially in the MCI group), we performed eight (for the eight experimental conditions; see Table 2) one-sample $t$ tests against the value 0 (which represents responses by chance), all of which were statistically significant (all $p<.01)$, indicating that both healthy and MCI groups (and their cognitive reserve subgroups) learned in some degree both explicit and implicit relationships between pairs of words.

The mixed ANCOVA $2 \times 2 \times 2$ on hits showed no significant main effects or interactions. Finally the same ANCOVA on false alarms only showed as only significant the interaction groups by tasks $(F(1,71)=$ $\left.4.94, p<.05, \eta^{2}=.07\right)$. Post-hoc analysis on this interaction showed that the healthy sample committed fewer false alarms in the E+I task than in the E task (means 0.41 and 0.52 , respectively; $t(38)=4.71, p<.0001)$, what improved its overall performance in the former task, whereas the MCI committed similar rates of false alarms in both tasks (means 0.51 and 0.53 , respectively; $t(36)<1$ ).

\section{Discussion}

This study's aim was to analyze implicit and explicit memory performance as a function of cognitive reserve and neuropsychological status. We selected two groups

Table 2. Mean proportions of hits (H), false alarms (FA) and hits minus $F A(H-F A)$ for the two recognition tests (E: explicit; E+I: explicit plus implicit) as a function of groups (Healthy vs MCI: Mild Cognitive Impairment) and cognitive reserve (CR: Low vs High)

\begin{tabular}{|c|c|c|c|c|c|c|c|c|c|c|c|c|}
\hline \multirow[b]{3}{*}{ Recognition test } & \multicolumn{6}{|c|}{ Healthy Controls } & \multicolumn{6}{|l|}{ MCI } \\
\hline & \multicolumn{2}{|c|}{ H-FA } & \multicolumn{2}{|l|}{$\mathrm{H}$} & \multicolumn{2}{|l|}{ FA } & \multicolumn{2}{|c|}{$\mathrm{H}-\mathrm{FA}$} & \multicolumn{2}{|l|}{$\mathrm{H}$} & \multicolumn{2}{|l|}{ FA } \\
\hline & $\mathrm{E}$ & $\mathrm{E}+\mathrm{I}$ & $\mathrm{E}$ & $\mathrm{E}+\mathrm{I}$ & $\mathrm{E}$ & $\mathrm{E}+\mathrm{I}$ & $\mathrm{E}$ & $\mathrm{E}+\mathrm{I}$ & $\mathrm{E}$ & $\mathrm{E}+\mathrm{I}$ & $\mathrm{E}$ & $\mathrm{E}+\mathrm{I}$ \\
\hline Low CR & 0.11 & 0.30 & 0.67 & 0.73 & 0.56 & 0.43 & 0.12 & 0.10 & 0.65 & 0.62 & 0.53 & 0.54 \\
\hline High CR & 0.28 & 0.40 & 0.75 & 0.76 & 0.47 & 0.35 & 0.08 & 0.14 & 0.58 & 0.63 & 0.50 & 0.49 \\
\hline
\end{tabular}


of participants: one healthy and one diagnosed with MCI. Both groups carried out an associative recognition task where success depended on respondents' ability to establish an explicit relationship in the first condition, or explicit and implicit relationships in the second condition. Healthy participants performed better in the implicit-plus-explicit condition than in the explicit condition. The high cognitive reserve subsample outperformed the low-reserve subsample in both conditions. Those effects were not significant in either MCI subsample, however. A marginally significant interaction effect was found, though, such that participants with MCI and higher cognitive reserve tended to perform better in the explicit-plus-implicit condition than in the explicit condition.

Healthy participants were able to acquire the implicit relationship embedded in the explicit connection in word pairs to increase their performance. This replicates similar results reported by our group in young and several elderly populations with and without health problems (see Algarabel et al., 2009; 2012; 2013; Algarabel \& Pitarque, 2010; Algarabel, Pitarque et al., 2010: Algarabel, Rodríguez et al., 2010). More importantly, we detected differential performance in healthy participants with high and low cognitive reserve in both explicit and implicit tests, results that agree those of Gordon et al. (2013), who found that cognitive reserve largely determines a person's level of priming (but see Reber et al., 1991).

However the results of the MCI group were quite dissimilar from those of the healthy control group. The absence of any effect of task type or cognitive reserve is a very conspicuous result, particularly given the positive effects of those variables usually reported in different populations (Algarabel et al., 2009, Algarabel, Rodríguez et al., 2010). Participants were not able to implicitly learn the connection between the two words in each word pair. Looking at differential performance in the healthy and MCI groups, something shocking comes to light: the healthy sample with low cognitive reserve and the two MCI groups performed similarly at baseline (explicit associative recognition), the former improved by a considerable margin under the implicitplus-explicit condition, whereas the MCI group did not. In conclusion, it seems MCI participants were deficient in perceptual processing of implicit relationships. This MCI-associated deficit could be reminiscent of the familiarity deficit reported in earlier studies (Algarabel et al., 2009, 2012; Algarabel, Rodríguez et al., 2010). In general, as indicated in the introduction (Dew et al., 2007; Fleischman \& Gabrieli, 1998; Fleischman et al., 2004; Gordon et al., 2013; Light \& Singh, 1987), healthy elderly adults display no priming or familiarity deficits on yes-no recognition tasks. In this case, though, contrary to previous reports, MCI participants did.
Despite these null effects in MCI subgroups, the interaction effect of task and cognitive reserve turned out to be marginally significant, indicating that MCI people with high CR tended to learn the implicit relationship. Probably with larger samples such interaction would have been significant. As discussed above (Stern, 2009), compensation can be expected in the early stages of cognitive decline, or when no behavioral consequences of pathology are evident. This is one of few studies to establish the cognitive reserve variable categorically, and examine its effect experimentally rather than through correlation. Given its nature we have attempted to separate as much as possible the CR groups, but that may not have been enough, particularly for MCI participants. Finally, we tried to give participants a relatively extensive training phase, but it may have been insufficient, particularly for the MCI sample. Further research must be conducted to either confirm or refute these potentially important results.

\section{References}

Alameda J. R., \& Cuetos F. (1995). Diccionario de frecuencias de las unidades lingüísticas del castellano [Frequency dictionary of the linguistic units of Spanish]. Oviedo, Spain: Universidad de Oviedo, Departamento de Psicología, Oviedo.

Algarabel S., \& Pitarque A., (2010). Familiarity changes as a function of perceptual shifts. The Spanish Journal of Psychology, 13, 518-524. http://dx.doi.org/10.1017/ S1138741600002213

Algarabel S., Escudero J., Mazón J. F., Pitarque A., Fuentes M., Peset V., \& La Cruz L. (2009). Familiarity-based recognition in the young, healthy elderly, mild cognitive impaired and Alzheimer's patients. Neuropsychologia, 47, 2056-2064. http://dx.doi.org/10.1016/j.neuropsychologia.2009.03.016

Algarabel S., Fuentes M., Escudero J., Pitarque A., Peset V., Mazón J. F., \& Meléndez J. C. (2012). Recognition memory deficits in mild cognitive impairment. Aging, Neuropsychology, and Cognition, 19, 608-619. http:/ /dx. doi.org/10.1080/13825585.2011.640657

Algarabel S., Pitarque A., Cómbita L. M., \& Rodríguez L. A. (2013). Implicit relational effects in associative recognition. Psicológica, 34, 163-177.

Algarabel S., Pitarque A., Tomás J. M., \& Mazón J. F. (2010a). Explorations on familiarity produced by words with specific combinations of letters. European Journal of Cognitive Psychology, 22, 265-285. http:/ /dx.doi.org/ $10.1080 / 09541440902767818$

Algarabel S., Rodríguez L. A., Escudero J., Fuentes M., Peset V., Pitarque A., \& Mazón J. (2010b). Recognition by familiarity is preserved in Parkinson's without dementia and Lewy-Body disease. Neuropsychology, 24, 599-607.

Angel L., Fay S., Bouazzaoui B., Baudouin A., \& Isingrini M. (2010). Protective role of educational level on episodic memory aging: An event-related potential study. Brain and Cognition, 74, 312-323. http://dx.doi.org/10.1016/ j.bandc.2010.08.012

Bender A. R., Naveh-Benjamin M., \& Raz N. (2010). Associative deficit in recognition memory in a lifespan 
sample of healthy adults. Psychology and Aging, 25, 940-948. http:/ /dx.doi.org/10.1037/a0020595

Benedet M. J., \& Alexandre M. A. (1998). Test de Aprendizaje Verbal España-Complutense (TAVEC) [Spain-Complutense Verbal Learning Test]. Madrid, Spain: TEA.

Colom R., Jung R. E., \& Haier R. J. (2006). Distributed brain sites for the g-factor of intelligence. NeuroImage, 31, 1359-1365. http:/ / dx.doi.org/10.1016/j.neuroimage.2006.01.006

Conway A. R. A., Kane M. J., \& Engle R. W. (2003). Working memory capacity and its relation to general intelligence. Trends in Cognitive Sciences, 7, 547-552. http:/ /dx.doi.org/ 10.1016/j.tics.2003.10.005

Craik F. I. M. (2002). Levels of processing: Past, present, and future?. Memory, 10, 305-318. http:/ /dx.doi.org/10.1080/ 09658210244000135

Craik F. I. M., \& Tulving E. (1975). Depth of processing and the retention of words in episodic memory. Journal of Experimental Psychology, 104, 268-294. http:/ /dx.doi.org/ 10.1037/0096-3445.104.3.268

Dew I. T. Z., \& Giovanello K. S. (2010). Differential age effects for implicit and explicit conceptual associative memory. Psychology and Aging, 25, 911-921. http:/ / dx.doi. org/10.1037/a0019940

Dew I. T. Z., Bayen U. J., \& Giovanello K. S. (2007). Implicit relational memory in young and older adults. Journal of Psychology, 215, 25-34. http://dx.doi.org/10.1027/ 0044-3409.215.1.25

Fleischman D. A., \& Gabrieli J. (1998). Repetition priming in normal aging and Alzheimer's disease: A review of findings and theories. Psychology and Aging, 13, 88-119. http:/ / dx.doi.org/10.1037/0882-7974.13.1.88

Fleischman D. A., Wilson R. S., Gabrieli J. D. E., Bienias J. L., \& Bennett D. A. (2004). A longitudinal study of implicit and explicit memory in old persons. Psychology and Aging, 19, 617-625. http://dx.doi.org/10.1037/0882-7974.19.4.617

Folstein M. F., Folstein S. E., \& McHugh P. R. (1975). Mini Mental State. A practical method for grading the cognitive state of patients for the clinical. Journal of Psychiatric Research, 12, 189-198. http:/ /dx.doi.org/10.1016/ 0022-3956(75)90026-6

Gordon L. T., Soldan A., Thomas A. K., \& Stern Y. (2013). Effect of repetition lag on priming of unfamiliar visual objects in young and older adults. Psychology and Aging, 28, 219-231. http://dx.doi.org/10.1037/a0030929

Graf P., \& Schacter D. L. (1985). Implicit and explicit memory for new associations in normal and amnesic subjects. Journal Experimental Psychology: Learning, Memory, and Cognition, 11, 501-518. http://dx.doi.org/10.1037/ 0278-7393.11.3.501

Light L. L., \& Singh A. (1987). Implicit and explicit memory in young and older adults. Journal of Experimental Psychology: Learning, Memory and Cognition, 13, 531-541. http:/ / dx.doi.org/10.1037/0278-7393.13.4.531

Lobo A., Saz P., \& Marcos G. (2002). Adaptación del Examen Cognoscitivo Mini-Mental [Adaptation of Mini-Mental State Examination]. Madrid, Spain: TEA Ediciones.

Old S. R., \& Naveh-Benjamin M. (2008). Differential effects of age on item and associative measures of memory: A meta-analysis. Psychology and Aging, 23, 104-118. http:/ /dx.doi.org/10.1037/0882-7974.23.1.104
Peña-Casanova J. (2005). Test Barcelona revisado [Revised Barcelona Test]. Barcelona, Spain: Masson.

Petersen R. C. (2004). Mild cognitive impairment as a diagnostic entitiy. Journal of Internal Medicine, 256, 183-194. http:/ / dx.doi.org/10.1111/j.1365-2796.2004.01388.x

Reber A. S., Walkenfeld F. F., \& Hernstadt R. (1991). Implicit and explicit learning: Individual differences and IQ. Journal of Experimental Psychology: Learning, Memory, and Cognition, 17, 888-896. http:/ /dx.doi.org/10.1037/0278-7393.17.5.888

Reingold E. M., \& Goshen-Gottstein Y. (1996). Automatic retrieval of new associations under shallow encoding conditions. Consciousness and Cognition, 5, 117-130. http:/ / dx.doi.org/10.1006/ccog.1996.0007

Reisberg B., Ferris S. H., de Leon M. J., \& Crook T. (1982). The Global Deterioration Scale for assessment of primary degenerative dementia. The American Journal of Psychiatry, 139, 1936-1939.

Rey A. (1999). Test de copia y reproducción de memoria de figuras geométricas complejas [Copy and Reproduction of Complex Geometric Figures from Memory Test]. Madrid, Spain: TEA editions.

Scarmeas N., \& Stern Y. (2003). Cognitive reserve and lifestyle. Journal of Clinical and Experimental Neuropsychology, 25, 625-633. http://dx.doi.org/10.1076/jcen.25.5.625.14576

Solé-Padullés C., Bartrés-Faz D., Junqué C., Vendrell P., Rami L., Clemente I. C., ..., \& Molinuevo J. L. (2009). Brain structure and function related to cognitive reserve variables in normal aging, mild cognitive impairment and Alzheimer's disease. Neurobiology of Aging, 30, 1114-1124. http:/ / dx.doi.org/10.1016/j.neurobiolaging.2007.10.008

Stern Y. (2009). Cognitive reserve. Neuropsychologia, 47, 2015-2028. http://dx.doi.org/10.1016/j. neuropsychologia.2009.03.004

Stern Y., Habeck C., Moeller J., Scarmeas N., Anderson K. E., Hilton H. J., ..., \& van Heertum R. (2005). Brain networks associated with cognitive reserve in healthy young and old adults. Cerebral Cortex, 15, 394-402. http:/ /dx.doi.org/ 10.1093/cercor/bhh142

Valenzuela M., \& Sachdev P. (2006). Brain reserve and dementia: A systematic review. Psychological Medicine, 36, 441-454. http:/ / dx.doi.org/10.1017/S0033291705006264

Vance D. E., \& Crowe M. (2006). A proposed model of neuroplasticity and cognitive reserve in older adults. Activities, Adaptation E Aging, 30, 61-79. http:/ /dx.doi. org/10.1300/J016v30n03_04

Wechsler D. (2004). Wechsler Memory Scale III. Madrid, Spain: TEA ediciones.

Wechsler D. (2001). Wechsler Intelligence Scale for Adults-III (WAIS-III). Madrid, Spain: TEA ediciones.

Yesavage J. A., Brink T. L., Rose T. L., Lum O., Huang V., Adey M., \& Leirer V. O. (1983). Development and validation of a geriatric depression scale: A preliminary report. Journal of Psychiatric Research, 17, 37-49. http:/ / dx.doi.org/10.1016/0022-3956(82)90033-4

Zahodne L. B., Glymour M. M., Sparks C., Bontempo D., Dixon R. A., MacDonald S. W. S., \& Manly J. J. (2011). Education does not slow cognitive decline with aging: 12-year evidence from the Victoria Longitudinal Study. Journal of the International Neuropsychological Society, 17, 1039-1046. http:/ /dx.doi.org/10.1017/S1355617711001044 